\title{
Low Molecular Weight Heparin Inhibits Circulating Fibrocytes Differentiation by Modulating Neuronal Nitric Oxide Synthase and TGF- $\beta 1 /$ Smad Pathway
}

\author{
Xudong Xie Liangfeng Zhu Zhongying Fu Xiaogang Guo Kan Wang Xiaosheng $\mathrm{Hu}$ \\ Junzhu Chen \\ Department of Cardiovascular Diseases, First Affiliated Hospital, School of Medicine, Zhejiang \\ University, Hangzhou, China
}

\section{Key Words}

Low molecular weight heparin • Circulating fibrocytes $•$ Fibrosis $•$ Nitric oxide synthase - Transforming growth factor $-\beta 1$

\begin{abstract}
Background/Aims: Circulating fibrocytes (CFs) have been placed at the center of a number of fibrosing conditions. Recently, attention has been drawn to the non-anticoagulant activities of low molecular weight heparin (LH), especially its anti-fibrotic effects. The purpose of this study was to investigate the effects of LH on CFs differentiation and possible underlying mechanisms. Methods/Results: CFs were cultured from human peripheral blood mononuclear cells and identified by dual-immunofluorescence staining. Incubation with LH inhibited CFs trans-differentiation by upregulating CD34 and downregulating pro-Collagen I and a-SMA in a concentration- and time-dependent manner, all of which were detected by flow cytometry. Similar effects were observed after incubation with L-NAME, an inhibitor of NOS. NO production was measured by Griess methods and markedly decreased in CFs treated with LH. Three NOS isoforms were assessed by western blot and nNOS was the predominant isoform involved in this process. Additionally, LH and L-NAME had similar down-regulating effects on the expression of TGF- $\beta 1$ and $\mathrm{pSmad} 2 / 3$, which indicated that TGF- $\beta / S$ mad pathway might be a downstream signaling of nNOS/NO during LH treatment. Conclusion: These results suggested that LH could exhibit anti-fibrotic effects by inhibiting CFs transdifferentiation, in which the involvement of $\mathrm{nNOS} / \mathrm{NO}$ and TGF- $\beta / S m a d$ pathway were identified.
\end{abstract}




\section{Introduction}

Circulating fibrocytes (CFs), a distinct population of blood-borne cells that co-express hematopoietic cell antigens and fibroblast products, have been shown to play an important role in the generation of fibrosis in different fibrosing disorders, such as renal fibrosis, pulmonary fibrosis and cirrhosis [1-3]. Studies have demonstrated that CFs traffic to the site of tissue injury, differentiate into fibroblasts and myofibroblasts, and contribute to collagen deposition and fibrosis [4-6]. However, the regulatory processes that govern the differentiation of blood-borne fibrocytes are currently not known.

Low molecular weight heparin (LH) is produced by the depolymerization of unfractionated heparin with lower mean $\mathrm{M}_{\mathrm{r}}$ between 3 and $6 \mathrm{kD}$ and chain lengths of 12 to 18 saccharide units. Despite their similar anticoagulant activities between LH and heparin, the structure differences give LH distinct non-anticoagulant properties. Recently, attention has been drawn to the anti-fibrotic effect of LH in both animal models and human diseases [7-10]. Additionally, studies in vitro have reported that LH contributes to the differentiation of several types of progenitor cells $[11,12]$.

Based on these studies, it was hypothesized that LH might act as anti-fibrotic agent via inhibiting CFs trans-differentiation into fibroblasts and myofibroblasts. We therefore investigated the effects of different concentrations of LH on CFs differentiation and the time course of the potential effects, and the underlying signal transduction pathways.

\section{Materials and Methods}

\section{Cell Culture and Stimulation}

CFs from human peripheral blood were isolated and characterized as previously described [13]. To harvest and culture human $\mathrm{CFs}, 20 \mathrm{ml}$ of venous blood was collected in a heparinized centrifuge tube (BD Biosciences) from healthy volunteers who had provided written informed consent. Peripheral blood mononuclear cells (PBMCs) were separated from whole blood by centrifugation on a Ficoll density gradient (Invitrogen, Carlsba, CA) following the manufacturer's potocol. After centrifugation at $2000 \mathrm{~g}$ for $20 \mathrm{~min}$, the cells at the interface were harvested, washed twice, and resuspended in DMEM (GIBICO, USA) containing $20 \%$ FCS (Sijiqing Biotech, China). After 5 days of culture, non-adherent cells were removed by gentle aspiration and media were replaced. Adherent cells were cultured continually for another 5-9 days for further experiments. One day before drug administration (10th day), cells were under serum starvation for $24 \mathrm{~h}$ in DMEM, 2\% FCS, released from growth arrest by adding DMEM, 20\% FCS, with various LH concentrations for various incubation times, then washed and used in next assays. CFs were divided into 5 groups. (1) Control group: DMEM containing 20\% FCS without LH; (2)-(5) LH treatment group: containing $0.1,1,10$ and $100 \mathrm{IU} / \mathrm{ml}$ LH respectively. L-NAME (1mM), a nitric oxide synthase (NOS) inhibitor, was used as the positive control. For time course assay, CFs were treated with the most effective agents for $12 \mathrm{~h}, 24 \mathrm{~h}$, $48 \mathrm{~h}$ and $72 \mathrm{~h}$, respectively.

The LH (FraxiParine, 4100 anti Xa IU/0.4mL) was purchased from Hangzhou Sanofi Minsheng limited companies.

\section{Dual immunofluorescence and confocal microscopy}

CFs were identified by means of dual immunofluorescence for CD34 and pro-collagen I (pro-ColI) expression. The cells seeded on fibronectin-coated chamber slides on 10th day were fixed with $4 \%$ paraformaldehyde for $5 \mathrm{~min}$ at $4{ }^{\circ} \mathrm{C}$ and permeabilized with $0.1 \%$ Triton X-100 for $20 \mathrm{~min}$ in PBS. The cells were incubated with a polyclonal goat anti-human pro-Coll antibody (Santa Cruz, dilution 1:50) overnight at $4^{\circ} \mathrm{C}$. The slides next were incubated for $1 \mathrm{~h}$ at room temperature with an Alexa Fluor 488-labeled rabbit anti-goat antibody (Molecular Probes, 1:100). Then the slides were incubated with a monoclonal mouse anti-human CD34 antibody (Santa Cruz, dilution 1:100) overnight at $4^{\circ} \mathrm{C}$ and then with an Alexa Fluor 546-labeled goat anti-mouse antibody (Molecular Probes, 1:100). This was followed by staining with DAPI (Sigma, 1:10000) for $20 \mathrm{~min}$. Normal goat or mouse IgG was used instead of primary antibody as negative 
Xie/Zhu/Fu et al.: Low Molecular Weight heparin Inhibits Circulating Fibrocytes Differentiation

control. Each step was followed by thorough washing with PBS. Confocal microscopy was performed using a fluorescence laser scanning confocal microscope (Olympus Fluoview FV 1000).

Flow cytometry

The cultured fibrocytes $\left(1 \times 10^{6}\right.$ cells $)$ were immersed in permeabilizing solution and incubated with antibodies against CD34, pro-col I and a-SMA (mouse monoclonal antibody, Dako), indirectly labeled with Alexa Fluor 488 or 546 as required. All experiments included isotype controls. Cells $(2 \times 105)$ were flow cytometrically analyzed using the Cell Quest software (Becton Dickinson) and expressed as the results.

\section{Determination of nitric oxide (NO) generation}

The level of nitrite/nitrate in the conditioned medium of CFs was measured using a commercial kit (Nanjing Jiancheng Biology Graduate School, P.R. China). In brief, $50 \mu \mathrm{l}$ of medium or standard $\mathrm{NaNO}_{2}$ was mixed with $50 \mu \mathrm{l}$ of Griess reagent in a 96-well plate. After $15 \mathrm{~min}$, optical density was read in a microplate reader at $540 \mathrm{~nm}$ with a spectrophotometer. Each experiment was performed in triplicate. A standard curve using $\mathrm{NaNO}_{2}$ was generated for each experiment for quantification. Results of six independent experiments were used for statistical analysis.

\section{Western blot}

The extracted cell protein of each sample (30 $\mu \mathrm{g}$ total protein) was fractionated by sodium dodecyl sulphate (SDS) polyacrylamide gel electrophoresis and transblotted onto nitrocellulose filters. The membranes were incubated overnight at $4^{\circ} \mathrm{C}$ using mouse monoclonal eNOS (Santa Cruz), mouse monoclonal iNOS (Transduction Laboratories), mouse monoclonal nNOS (Transduction Laboratories), rabbit polyclonal TGF- $\beta 1$ (Beijing Zhongshan), goat polyclonal Smad2/3 (Santa Cruz), rabbit polyclonal phosphor-Smad2/3(Santa Cruz) and mouse monoclonal $\beta$-actin (Sigma) antibodies. The membranes were then incubated for $2 \mathrm{~h}$ in the appropriate diluted secondary antibody. Signal was detected using an electrochemiluminescence (ECL) method. Exposed films were quantified on an automated imaging system (Eastman Kodak ${ }^{\mathrm{T}}$, Rochester, NY, USA). A molecular weight marker (Invitrogen ${ }^{\mathrm{TM}}$, Carlsbad, CA, USA) was used to estimate the molecular weight.

\section{Statistical analysis}

Data are expressed as the means \pm SEM. When analyzing three or more group, statistical significance was measured by one-way ANOVA. Student's t test was performed to compare two groups. P values less than 0.05 were considered significant.

\section{Results}

\section{CFs morphology and identification}

When cultured in vitro, CFs gradually displayed an adherent, spindle-shaped morphology by adoption of a plump fibroblast-like appearance. Circular or oval nucleus was observed in the center of the cells as well as filopodia of different length at both ends. CFs were identified as fibroblast-like cells double positive for CD34 and pro-ColI (Fig. 1 A-D).

When incubated with LH, CFs exhibited distinct cellular morphologies compared with control. Increasing concentrations of LH inhibited CFs transformation into fibroblast-like cells and led to a more rounded, less mature appearance (Fig 1. F). Meanwhile, the number of CFs under LH treatment did not differ significantly from controls (all $p>0.05$ ).

\section{Effects of LH on CFs differentiation}

The concentration of $1 \mathrm{IU} / \mathrm{mL}$ was selected for time course analysis. It showed that the effect of LH on CFs was time-dependent. It reached to climax after $48 \mathrm{~h}(p<0.05)$ and presented decreasing tendency after $72 \mathrm{~h}$, but there was no significant difference between $48 \mathrm{~h}$ and $72 \mathrm{~h}(p>0.05)$.

We detected CFs phenotype by flow cytometry using the hemopoietic stem cell antigen CD34, the fibroblast marker pro-ColI and the myofibroblast marker a-SMA. After 
Fig. 1. Representative photomicrographs of cultured fibrocytes morphology. A-D, Fibrocytes were identified by dualimmunofluorescence staining using CD34 and pro-Collagen I. The separate markers for CD34 (red), pro-Collagen I (green) and nuclei staining (blue), and a merge picture (yellow) are shown. E-F, After 14 days in culture, more attached mononuclear cells displayed a spindle-shaped morphology (E). The LH treatment (10 IU/ml $\times 48 \mathrm{~h}$ ) could reduce the fibroblast-like change (F). Original magnification $\times 400(A-D)$ and $\times 200$ $(\mathrm{E}, \mathrm{F})$.
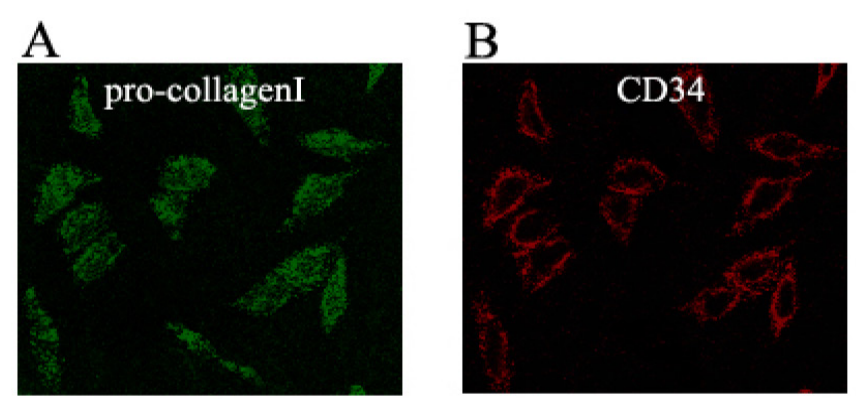

C

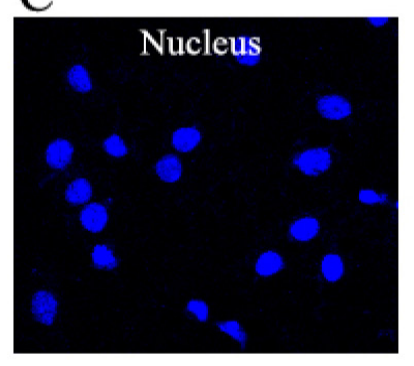

D

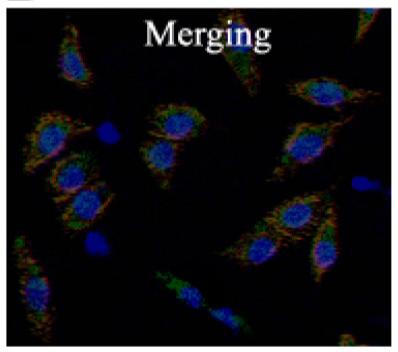

$\mathrm{E}$

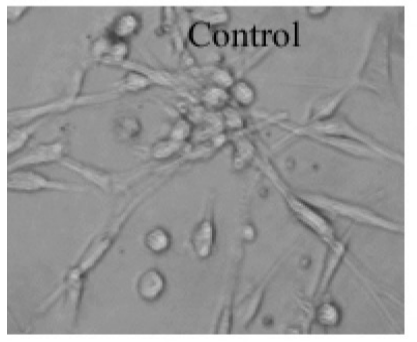

$\mathrm{F}$

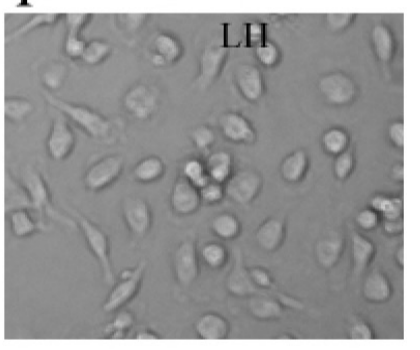

$48 \mathrm{~h}$ incubation with a range of $\mathrm{LH}$ concentrations, CFs differentiation was obviously suppressed in a dose-dependent manner in vitro. The percentages of CD34+ cells were increased significantly by $34.6 \%, 52.7 \%$ and $48.8 \%$, respectively, after incubation with LH at concentrations of $1,10,100 \mathrm{IU} / \mathrm{ml}$ (all $p<0.05$ or 0.01 ) (Fig. 2). Conversely, there was a dosedependent decrease in the number of CFs stained positively for pro-Coll, with a maximal decline of $34 \%$ in response to $10 \mathrm{IU} / \mathrm{ml}$ compared with the controls (all $p<0.05$ or 0.01 )(Fig. 2). Similar results were observed in the percentage of a-SMA ${ }^{+}$cells after treated with $\mathrm{LH}$, showing decreases of approximately $28 \%, 34 \%$ and $33 \%$ ( all $p<0.05$ or 0.01 ), respectively, compared with controls (Fig. 2).

As previous studies had established the notion that NO and its NOS isoforms were involved in the differentiation of stem/progenitor cells, in the current study, we have investigated the effects of L-NAME, a direct inhibitor of NOS, on CFs differentiation. Interestingly, all the three marker proteins displayed an almost similar expression pattern in L-NAME group, showing no significant difference from LH group at concentrations of 1 , 10 or $100 \mathrm{IU} / \mathrm{ml}$ (all $p>0.05$ ), which indicated that NOS/NO might be involved in LH-driven inhibition of CFs differentiation.

\section{Effects of LH on NOS expression and NO production from CFS}

Treatment with LH resulted in a dose-dependent reduction in the NO production from CFs (Fig. 3). A significant decline was reached at $10 \mathrm{IU} / \mathrm{ml}$ and sustained at $100 \mathrm{IU} / \mathrm{ml}$ compared with the controls (both $p<0.01$ ), but no difference was observed between the two groups. The NO level either in 10 or in $100 \mathrm{IU} / \mathrm{ml}$ group was similar with L-NAME group. 


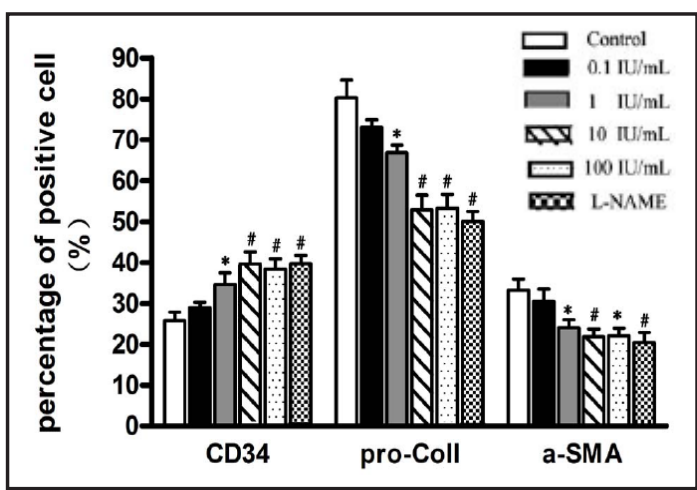

Fig. 2. Effects of $\mathrm{LH}$ on the CFs differentiation. The percentages of CD34+, pro-Collagen I+ and a-SMA+ cells were detected by flow cytometry after CFs were treated for $48 \mathrm{~h}$ with different concentrations of 0 , $0.1,1,10,100 \mathrm{IU} / \mathrm{ml} \mathrm{LH}$ or $1 \mathrm{mM} \mathrm{L-NAME}{ }^{*} p<0.05$; $\# p<0.01$.

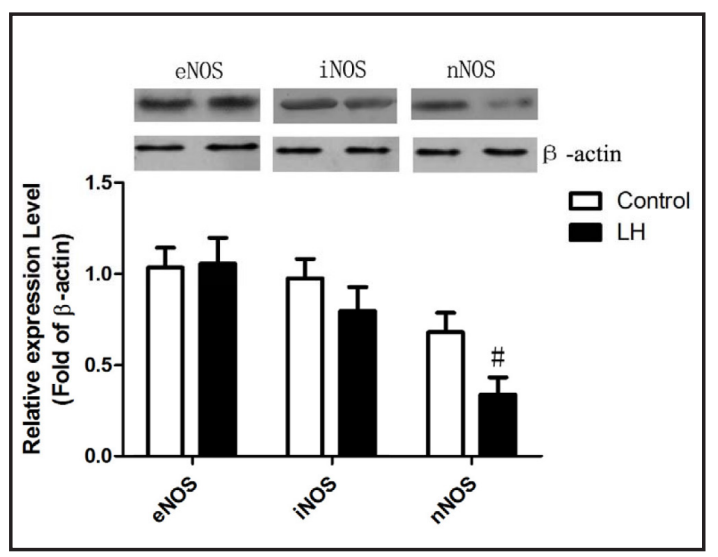

Fig. 4. Effects of LH on eNOS, iNOS and nNOS protein expression in CFs. Western blot analysis were performed after CFs were treated for $48 \mathrm{~h}$ with 10 $\mathrm{IU} / \mathrm{ml} \mathrm{LH}$. Normalization of expression levels was done by comparison with $\beta$-actin. Data are shown as mean $\pm \operatorname{SEM}(\mathrm{n}=5)$. \# $p<0.01$.

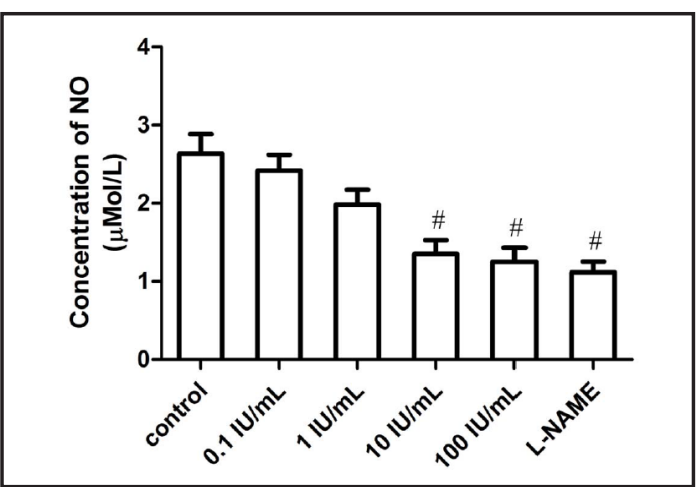

Fig. 3. Effects of LH on the NO generation from CFs. NO production was assayed by the Griess method in CFs treated for $48 \mathrm{~h}$ with $\mathrm{LH}$ at the concentrations of 0, 0.1, 1, 10 and $100 \mathrm{IU} / \mathrm{ml}$, or $1 \mathrm{mM} \mathrm{L-NAME.} \mathrm{Data}$ are expressed as the mean \pm SEM $(\mathrm{n}=6)$. \# $p<0.01$.

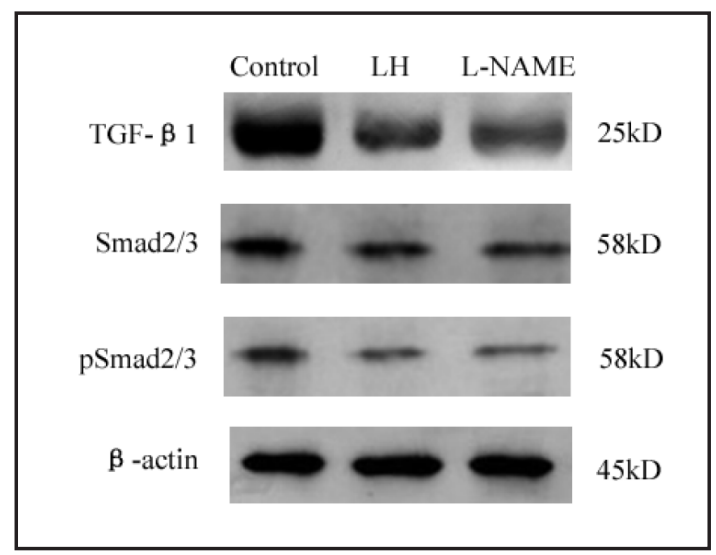

Fig. 5. Effects of LH on TGF- $\beta, \operatorname{Smad} 2 / 3$ and phosphor-Smad2/3 protein expression in CFs. Western blot analysis were performed after CFs were treated for $48 \mathrm{~h}$ with $10 \mathrm{IU} / \mathrm{ml} \mathrm{LH}$ or $1 \mathrm{mM} \mathrm{L}$-NAME. $\beta$-actin was used as a loading control.

We next identified the NOS isoforms expressions in CFs. As shown in Fig. 4, the protein expressions of all three isoforms (eNOS, iNOS and nNOS) were identified in CFs by western blot and densitometric analysis showed significantly higher levels of eNOS and iNOS compared with nNOS (both $p<0.05)$. After treated with LH $(10 \mathrm{IU} / \mathrm{ml}$ for $48 \mathrm{~h}$ ), the level of nNOS was decreased about 50\% ( $p<0.01)$, whereas no significant differences were detected in eNOS and iNOS under the same incubation conditions. These results suggested that nNOS was likely the amenable isoform involved in the LH-driven suppression of CFs differentiation.

\section{LH treatments down-regulate TGF- $\beta 1 /$ Smad expression}

TGF- $\beta 1$ is an important fibrogenic and growth-regulating cytokine. The present study further investigated whether TGF- $\beta 1 /$ Smad pathway was regulated downstream of nNOS/ NO in LH-mediated anti-fibrotic effect. Fig. 5 showed that LH (10 IU/ml for $48 \mathrm{~h}$ ) significantly reduced the contents of TGF- $\beta 1$ and pSmad2/3 compared with controls (both $p<0.05$ ), similar to the L-NAME group. 


\section{Discussion}

To the best of our knowledge, the present study described for the first time that LH could exert its potent anti-fibrotic activities by inhibiting CFs differentiation into fibroblasts and myofibroblasts in a dose- and time-dependant manner. LH-induced suppression of CFs tras-differentiation was associated with the inhibition of nNOS/NO signaling, as evidenced by the obvious decrease in nNOS expression and subsequent NO production. Furthermore, TGF- $\beta 1$ and pSmad were markedly reduced after LH stimulation and similar results were obtained from L-NAME treatment, which indicating that TGF- $\beta 1 /$ Smad pathway might be involved in the downstream of nNOS/NO signaling in LH antifibrotic effects.

It was found that LH had many potential therapeutic benefits beyond its traditional role as an anticoagulant. Recently, both experimentally and clinically its anti-fibrotic properties have attracted particular attention. LH has been reported to have beneficial effects on inflammation and collagen deposit in several animal models [7, 8]. Moreover, clinical data suggested that LH was protective against liver fibrosis in patients with chronic hepatitis [9, 10]. However, although LH has been shown to have protective effects against fibrogenesis, the underlying mechanisms for the anti-fibrotic effects are far from being understood.

Fibrocytes are circulating bone marrow-derived progenitors that co-express haematopoietic stem-cell antigens, markers of the monocyte lineage and fibroblast products. There has been significant evidence from across different subspecialties of medicine implicating these cells in different tissue-specific or organ-specific fibrosing disorders [1-3]. CFs comprise $0.1-0.5 \%$ of non-erthrocytic cells in peripheral blood and display an adherent, spindle-shaped morphology when cultured in vitro, which consistent with our study [14]. $\mathrm{CD}^{+} 4^{+}$fibrocytes undergo morphological and phenotypic alterations characterized by the adoption of a plump fibroblast-like appearance and loss of CD34+ expression accompanied by a gain of a-SMA expression [15]. In our study, we found LH exerted a pronounced inhibitory effect on CFs differentiation into fibroblasts and myofibroblasts, reserving its haematopoietic stem cells phenotype (CD34+). Fibroblasts have been shown to exhibit increased collagen expression and other matrix components in certain fibrotic disease states. Myofibroblasts are the cell type that generates and deposits collagen rich pathological extracellular matrix leading to irreversible fibrosis and causing organ dysfunction. There is increasing evidence that CFs contribute to the new population of myofibroblasts that emerge at the tissue site during the process of fibrosis $[5,6,15]$. Moreover, studies in vitro have reported that LH contributed to modulating the differentiation of progenitor cells, such as endometrial stromal cells and osteoblast $[11,12]$. Taken together, these findings suggest that LH may function as anti-fibrotic agent by strong inhibition of CFs differentiation, which supporting the potential therapeutic benefits of LH in fibrosing diseases.

NO has been widely studied due to its significant importance to human cell physiology, including cell proliferation and differentiation. NO is synthesized from L-arginine by three isoforms of NOS: neuronal NOS (nNOS), inducible NOS (iNOS), and endothelial NOS (eNOS). In our previous study, it was demonstrated that eNOS/NO was involved in the Ang(1-7) induced suppression of CFs proliferation and apoptosis [13]. But there is a dearth of studies regarding all NOS isoforms expression pattern in CFs. In the present study, we identified all three NOS isoforms in CFs by western blot and relatively higher expression of eNOS and iNOS. Recently, NO has been shown to play a protagonist role in the therapeutic benefits of heparin and LH. Lakshimi et al reported that LH would be more effective in the treatment of burn patients through NOS inhibition [16]. Another report also showed that heparin significantly ameliorated lung injury via the inhibition of NOS expression [17]. In this study, we found that LH significantly reduced the expressions of nNOS and subsequent NO production in cultured CFs. Moreover, as for the effects of L-NAME (a NOS inhibitor) treatment, blockade of NO biosynthesis could suppress the CFs differentiation in a compatible pattern such as observed in LH treated groups. These results confirmed previous reports demonstrating that reduction in NO production resulted in an increased number of early haematopoietic 
progenitors in nNOS knockout mice $[18,19]$. It had also been demonstrated that exposure to chemical donors of NO could influence the extent of CD34+ bone marrow cells maturation in vitro [20]. Thus nNOS/NO may be acting as a negative regulator of haematopoietic progenitor cells differentiation [21]. Taking all these results together, it is concluded that nNOS may be the predominant isoform involved in LH-induced inhibition of CFs differentiation toward the fibroblast or myofibroblast phenotype. Nevertheless, the precise mechanism by which LH inhibits the nNOS/NO pathway needs to be further elucidated.

TGF- $\beta 1$, a central mediator of fibrogenesis, has been shown to be the most important cytokine for the trans-differentiation of fibroblasts to myofibroblasts that exhibit increased aSMA staining, elevated collagen secretion, and increased stress fibers [22]. TGF- $\beta 1$ stimulates collagen synthesis via the phosphorylation of Smad 2 and Smad 3 [23]. Downregulation of TGF- $\beta 1$ expression and modulation of TGF- $\beta /$ Smad signaling may be effective in preventing organ fibrosis [24]. Investigators have previously demonstrated that TGF- $\beta 1$ overexpression has been associated with enhanced myofibroblast differentiaon $[25,26]$. In our data it was observed that the expressions of TGF- $\beta 1$ and p-Smad2/3 were markedly decreased in cultured CFs under LH treatment. These findings were consistent with a recent report which demonstrated that LH showed anti-fibrotic effect via inhibition of the TGF- $\beta$ / Smad pathway [15]. Moreover, the present study revealed a similar expression profile for TGF- $\beta 1$ and p-Smad2/3 between LH and L-NAME treatment group. In such contexts, it is possible to suggest that TGF- $\beta /$ Smad pathway may be a downstream signaling of nNOS in LH- stimulated CFs.

In conclusion, the present study shows a part of the mechanism of how LH exhibits anti-fibrotic effects in fibrogenesis. These data suggest for the first time that LH inhibits CFs differentiation through suppression of nNOS and its production NO, accompanied with down-regulation of TGF- $\beta /$ Smad pathway. Further studies are necessary to identify the molecules involved in modulation between LH and fibrocytes that are required for fibrocytes maturation.

\section{Acknowledgements}

This work was supported by the funds from National Natural Science Foundation of China (No.30900614 and No.81070082), Zhejiang Provincial Administration of traditional Chinese Medicine (No. 2009CB037).

\section{References}

1 Kisseleva T, Uchinami H, Feirt N, Quintana-Bustamante O, Segovia JC, Schwabe RF, Brenner DA: Bone marrow-derived fibrocytes participate in pathogenesis of liver fibrosis. J Hepatol 2006;45:429-438.

2 Moeller A, Gilpin SE, Ask K, Cox G, Cook D, Gauldie J, Margetts PJ, Farkas L, Dobranowski J, Boylan C, O'Byrne PM, Strieter RM, Kolb M: Circulating Fibrocytes Are an Indicator for Poor Prognosis in Idiopathic Pulmonary Fibrosis. Am J Respir Crit Care Med 2009;179:588-594.

-3 Wada T, Sakai N, Matsushima K, Kaneko S: Fibrocytes: a new insight into kidney fibrosis. Kidney Int 2007;72:269-273.

4 Bucala R: Circulating fibrocytes: cellular basis for NSF. J Am Coll Radiol 2008;5:36-39.

5 Mori L, Bellini A, Stacey MA, Schmidt M, Mattoli S: Fibrocytes contribute to the myofibroblast population in wounded skin and originate from the bone marrow. Exp Cell Res 2005;304:81-90.

6 Abu El-Asrar AM, Struyf S, Van Damme J, Geboes K: Circulating fibrocytes contribute to the myofibroblast population in proliferative vitreoretinopathy epiretinal membranes. Br J Ophthalmol 2008;92:699-704.

7 Lee JH, Lee H, Joung YK, Jung KH, Choi JH, Lee DH, Park KD, Hong SS: The use of low molecular weight heparin-pluronic nanogels to impede liver fibrosis by inhibition the TGF- $\beta$ /Smad signaling pathway. Biomaterials 2011;32:1438-1445. 


\section{Cellular Physiology $\quad$ Cell Physiol Biochem 2012;30:1465-1472

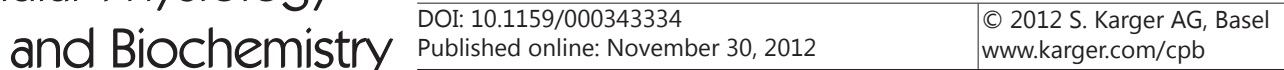

Xie/Zhu/Fu et al.: Low Molecular Weight heparin Inhibits Circulating Fibrocytes Differentiation

8 Frizelle S, Schwarz J, Huber SA, Leslie K: Evaluation of the effects of low molecular weight heparin on inflammation and collagen deposition in chronic coxsackievirus B3-induced myocarditis in A/J mice. Am J Pathol 1992;141:203-209.

-9 Bechmann LP, Sichau M, Wichert M, Gerken G, Kröger K, Hilgard P: Low-molecular-weight heparin in patients with advanced cirrhosis. Liver Int 2011;31:75-82.

10 Huang JS, Luo X, Yu JX, Liu W, Chen XW, Xie L, Zhu XY, Chen GY: Indigenous and imported low molecular weight heparin in the treatment of chronic hepatitis B and cirrhosis with hepatitis B virus: a prospective randomized controlled clinical study. Zhongguo Wei Zhong Bing Ji Jiu Yi Xue 2007;19:408-411.

11 Fluhr H, Spratte J, Ehrhardt J, Steinmüller F, Licht P, Zygmunt M: Heparin and low-molecular-weight heparins modulate the decidualization of human endometrial stromal cells. Fertil Steril 2010;93:25812587.

12 Yang L, Butcher M, Simon RR, Osip SL, Shaughnessy SG: The effect of heparin on osteoblast differentiation and activity in primary cultures of bovine aortic smooth muscle cells. Atherosclerosis 2005;179:79-86.

13 Wang K, Hu X, Du C, Tu S, Zhang F, Xie X: Angiotensin-(1-7) suppresses the number and function of the circulating fibrocytes by upregulating endothelial nitric oxide synthase expression. Mol Cell Biochem 2012;365:19-27.

14 LaPar DJ, Burdick MD, Emaminia A, Harris DA, Strieter BA, Liu L, Robbins M, Kron IL, Strieter RM, Lau CL: Circulating fibrocytes correlate with bronchiolitis obliterans syndrome development after lung transplantation: a novel clinical biomarker. Ann Thorac Surg 2011;92:470-477.

15 Kao HK, Chen B, Murphy GF, Li Q, Orgill DP, Guo L: Peripheral blood fibrocytes: enhancement of wound healing by cell proliferation, re-epithelialization, contraction, and angiogenesis. Ann Surg 2011;254:10661074.

16 Lakshmi RT, Priyanka T, Meenakshi J, Mathangi KR, Jeyaraman V, Babu M: Low molecular weight heparin mediated regulation of nitric oxide synthase during burn wound healing. Ann Burns Fire Disasters 2011;24:24-29.

17 Mu E, Ding R, An X, Li X, Chen S, Ma X: Heparin attenuates lipopolysaccharide-induced acute lung injury by inhibiting nitric oxide synthase and TGF- $\beta /$ Smad signaling pathway. Thromb Res 2012;129:479-485.

18 Krasnov P, Michurina T, Packer MA, Stasiv Y, Nakaya N, Moore KA, Drazan KE, Enikolopov G: Neuronal nitric oxide synthase contributes to the regulation of hematopoiesis. Mol Med 2008;14:141-149.

19 Epperly MW, Cao S, Zhang X, Franicola D, Shen H, Greenberger EE, Epperly LD, Greenberger JS: Increased longevity of hematopoiesis in continuous bone marrow cultures derived from NOS1 (nNOS, mtNOS) homozygous recombinant negative mice correlates with radioresistance of hematopoietic and marrow stromal cells. Exp Hematol 2007;35:137-145.

20 Shami PJ, Weinberg JB: Differential effects of nitric oxide on erythroid and myeloid colony growth from CD34+ human bone marrow cells. Blood 1996;87:977-982.

21 Michurina T, Krasnov P, Balazs A, Nakaya N, Vasilieva T, Kuzin B, Khrushchov N, Mulligan RC, Enikolopov G: Nitric oxide is a regulator of hematopoietic stem cell activity. Mol Ther 2004;10:241-248.

22 Biernacka A, Dobaczewski M, Frangogiannis NG: TGF- $\beta$ signaling in fibrosis. Growth Factors 2011;29:196202.

23 Yu L, Lin Q, Liao H, Feng J, Dong X, Ye J: TGF- $\beta 1$ induces podocyte injury through Smad3-ERK-NF- $\kappa B$ pathway and Fyn-dependent TRPC6 phosphorylation. Cell Physiol Biochem 2010;26:869-878.

24 Hawinkels LJ, Ten Dijke P: Exploring anti-TGF- $\beta$ therapies in cancer and fibrosis. Growth Factors 2011;29:140-152.

25 Gu L, Zhu YJ, Yang X, Guo ZJ, Xu WB, Tian XL: Effect of TGF-beta/Smad signaling pathway on lung myofibroblast differentiation. Acta Pharmacol Sin 2007;28:382-391.

26 Aoyagi-Ikeda K, Maeno T, Matsui H, Ueno M, Hara K, Aoki Y, Aoki F, Shimizu T, Doi H, Kawai-Kowase K, Iso T, Suga T, Arai M, Kurabayashi M: Notch Induces Myofibroblast Differentiation of Alveolar Epithelial Cells via Transforming Growth Factor-\{beta\}-Smad3 Pathway. Am J Respir Cell Mol Biol 2011;45:136-144. 